\title{
Smart information desk system with voice assistant for universities
}

\author{
Dabiah Alboaneen, Dalia Alsaffar, Amani Alqahtani, Lama Alamri, Amjad Alfahhad, \\ Bashaier Alqahtani, Alyah Alateeq, Rahaf Alamri, Fatimah Almohammedsaleh \\ Computer Science Department, College of Science and Humanities, Imam Abdulrahman Bin Faisal University, \\ Jubail, Kingdom of Saudi Arabia
}

\begin{tabular}{l} 
Article Info \\
\hline Article history: \\
Received May 19, 2020 \\
Revised Apr 7, 2021 \\
Accepted Apr 26, 2021 \\
\hline
\end{tabular}

\section{Keywords:}

Raspberry Pi

Smart mirror

Voice recognition

\begin{abstract}
This article aims to develop a smart information desk system through a smart mirror for universities. It is a mirror with extra capabilities of displaying answers for academic inquiries such as asking about the lecturers' office numbers and hours, exams dates and times on the mirror surface. In addition, the voice recognition feature was used to answer spoken inquiries in audio responds to serve all types of users including disabled ones. Furthermore, the system showed general information such as date, weather, time and the university map. The smart mirror was connected to an outdoor camera to monitor the traffics at the university entrance gate. The system was implemented on a Raspberry Pi 4 model B connected to a two-way mirror and an infrared (IR) touch frame. The results of this study helped to overcome the problem of the information desk absence in the university. Therefore, it helped users to save their time and effort in making requests for important academic information.
\end{abstract}

This is an open access article under the CC BY-SA license.

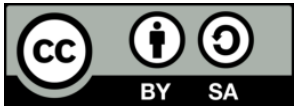

\section{Corresponding Author:}

Dabiah Alboaneen

Computer Science Department

College of Science and Humanities

Imam Abdulrahman Bin Faisal University

P.O. Box 31961, Jubail, Kingdom of Saudi Arabia

Email: dabuainain@iau.edu.sa

\section{INTRODUCTION}

In recent times, the developments of devices and intelligent interaction with devices have become very common. From that development, the concept of the internet of things (IoT) has emerged, which allows devices to communicate with each other in different and important places at the same time. Smart things are developed in ways that interact with each other, such as smart doors, smart homes and smart mirrors. Smart mirrors are intelligent devices that work as a mirror with the ability to interact with users and display various responses and information such as date, time and weather on a screen, which is behind a reflective surface that acts as a mirror. Human interaction with devices occurs in many ways, including gestures, voice and touch screen.

Today, there is a need for the academic environment to communicate smart in providing the services for students and faculty members. Students are now effectively relying on technology to communicate with each other and perform their different tasks more quickly than ever before. It is important to help students and save their time, and to make the university's resources available quickly and easily with high access. Many new students would like to know about the university during the first days of study through a tailored map showing them the different doors, exits and paths of the university. Some students also want to inquire 
about a lecturer or the number of the hall in which they are lectured. They may inquire from one of their colleagues about the lecturers' contact information or they may check their lecturers' offices by themselves, which will waste a lot of time and effort.

The motivation behinds this study is the absence of information desk in the university, which makes the students spend time and effort to get the information they need. In addition, the lack of services provided to people with special needs in the university, such as people with vision impairment. Hence, make use of the mirror in a beneficial way during the time spent in front of it with several ways to interact, such as the auditory method, by using voice recognition feature.

From this point of view, the aim is to develop a smart information desk system which provides services about student's queries regards the instructors, offices, classes and so on. The process of acquiring information from this system is done through a smart mirror that interacts with the users via a touch screen. The smart mirror displays date, time, weather, the latest announcements and a map of the university. In addition, the mirror provides real time images taking by a camera that monitors the university's entrance from the outside. It also allows the users to browse the university's website. These services will help to facilitate accessing the information that users need within the academic environment.

In summary, the key contributions of this article are as:

- Developing an information desk system with voice assistant to be utilized in the universities.

- Implementing the system through a smart mirror connected to a Raspberry Pi 4 model B, which makes this article, as the best of our knowledge, the first study that aims to provide a smart mirror as an information desk system for universities.

The remainder of this article is arranged as follows: Section 2 presents the related work on applications of smart mirrors. Section 3 describes the methods used in this study. The proposed smart mirror is presented in section 4. Finally, section 5 draws the conclusion and points out possible future work.

\section{RELATED WORK}

Smart mirrors have been widely implemented in the literature for different purposes. a review upon the applications of smart mirrors is presented in [1]. In [2]-[5], a smart mirror with wide application range was developed using a Raspberry Pi and it displays the weather, road traffic, news and user's schedules. Moreover, additional features such as face recognition, voice playback, remote control, Wi-Fi connectivity and a clothing indicator were included on a plasma screen in [3].

Voice recognition technology that allows the users to interact with the system via voice commands has also utilized in [6]-[8]. Hossain et al. [9] have focused on designing a smart mirror that facilitates controlling home devices and it verifies users' identities through face recognition technology. The mirror also displays general information such as time, weather and news.

Smart mirrors have also been used in the medical field to help in diagnosing the health condition of people. In [10]-[13], the authors have discussed how to use a smart mirror to detect facial expressions and signs to discover the health condition of a person. Erazo et al. [14] have developed a magic mirror that aims to support patients. Generally, the results proved that the patients accepted the developed system for the applied functionalities.

Furthermore, implementing smart mirrors in fashion and sport fields is getting popular. In [15]-[18], a smart mirror was made as a virtual fashion consultant, which can analyze, estimate and recommend the appropriate wearing and outfits by providing augmented reality (AR) and gestures recognition for body pose tracking and allowing the users to choose clothes in 2D visualization. However, Rahman et al. [19] and Iwabuchi et al. [20] aim to provide a smart makeup mirror that gives the users the ability to apply different makeup styles on their faces virtually using 3D visualization.

The study [21] aims to interactively support fitness and wellness exercises for the visitors of a touris- tic resort. The physical state of the users is evaluated by a technology-reinforced mirror, which consists of interactive home controller, AR and multimedia player. Besserer et al. [22], the smart mirror, FitMirror, was created as an interactive device that aims to improve the users mood by motivating them to wake up in the morning and do their exercises. The user can communicate with the system through a touch interface or voice commands.

Moreover, the academic field has also benefited from this innovation as smart mirrors started to be introduced in this filed for educational purposes such as teaching and simplifying the delivered concepts. The objective in [23] is to use the mirror in teaching anatomy for easier understanding. In addition, a camera is provided to track body poses by AR. Moreover, when the user stands in front of the camera, it creates perceptions and fantasies through the system besides 3D models for the body organs, text information and images about anatomy. 
Yang et al. [24] have developed a smart mirror to adjust and record users moods through recognizing their face expressions. Due to the impact of colors on the value of things to people, the mirror was tested by a number of university students. The students' comments varied between negative and positive on the efficiency of the mirror in identifying emotions correctly.

This study [25] aims to provide education and entertainment for the public in which a smart mirror connected to a camera and a screen is used in museums, science centers and other public places. As the virtual world is important in some applications such as scientific experiments, the mirror allows the users to see a reflection of themselves in addition to virtual objects provided using an AR system and transparency.

In contrast to these studies, the mirror developed in our study is intended to be used in the academic field as a source of information in the academic institution where it will be deployed. Moreover, the mirror has been connected to a customized information system developed to handle the users queries related to that institution which makes it distinguished from existing general purposes voice artificial intelligence (AI) systems.

\section{METHOD}

\subsection{Raspberry Pi}

The latest version, which is Raspberry Pi 4 Model B, is used. It is characterized by speed, high performance and high-energy efficiency. Raspberry Pi 4 has $1.5 \mathrm{GHz}$ processor and $4 \mathrm{~GB}$ of RAM for high efficiency. In addition, it has a Gigabit Ethernet, wireless and Bluetooth for fast communication.

\subsection{Light emitting diode (LED) monitor}

A LED monitor is used as the primary display device in our smart mirror in which it is connected to the Raspberry Pi via a high definition media interface (HDMI) cable. The monitor is used for displaying the system, which is installed in the Raspberry Pi. The LED monitor from class pro has the following specifications: screen size: 50", display with resolution (MP): 1920 x 1080, full high definition (FHD), it can be connected to the Internet via a Wi-Fi port and it has 3 HDMI ports.

\subsection{Mirror}

A two-way mirror is used that has two different surfaces, which are: a reflective surface that acts as a real mirror and a transparent surface that can display information. The mirror is custom made with a size of 50 " to meet our requirements.

\subsection{Camera and mini microphone}

A camera is used to monitor and keep tracking of the status of the traffic outside the university. The characteristics of the camera are: the C3W (ezGuard), a wall-mounted Full HD 1080p outdoor Wi-Fi camera, and capable of night vision. This camera offers two-way talking capability and stability in all weather conditions with a 360 swivel and foundation. A mini microphone is used to activate the voice recognition feature in the smart mirror, which allows it to respond to voice commands from users. It is connected to the Raspberry Pi via USB port.

\subsection{Infrared frames (IR-Frames)}

An IR-frame is used to activate the touch feature in the smart mirror as it provides a touch interface. It has IR sensors that are connected to the Raspberry Pi via USB port. We used model GT-IR-TB50, which is a recent version of IR-frame from GreenTouch. It has the following specifications: 50" IR touch overlay frame (10-touch points), active area size: 1,097 (H)x617.5 (V) $\mathrm{mm}$, each piece of the frame has its own LED set and each piece is connected with a 60-volt wire.

\subsection{Frame}

A wood frame, which is custom-designed with a size of 50" and a height of $1 \mathrm{~m}$ from the floor, is used to make the smart mirror looks like a normal mirror. Moreover, it works to cover and protect the Raspberry Pi with all the parts connected to it that will be placed behind the mirror.

\section{THE PROPOSED INFORMATION DESK SYSTEM}

\subsection{System implementation}

As shown in Figure 1, the hardware part of the system consists of a two-way mirror, LED monitor and a Raspberry Pi 4 model B. The process of building the smart mirror went through four steps as follows: 
1. The front and back sides of the mirror have been constructed as a wood frame that will be around the twoway mirror and work as a cover to protect the Raspberry Pi with all the parts connected to it which will be placed behind the mirror. Then, the wood planks have been fastened together to finish the wood frame construction.

2. In Figure 2, the two-way mirror and the LED monitor have been placed in the constructed wood frame. The two-way mirror is used such that it is semi-transparent acting as a mirror when it is inactive, and a glass window when information is being displayed.
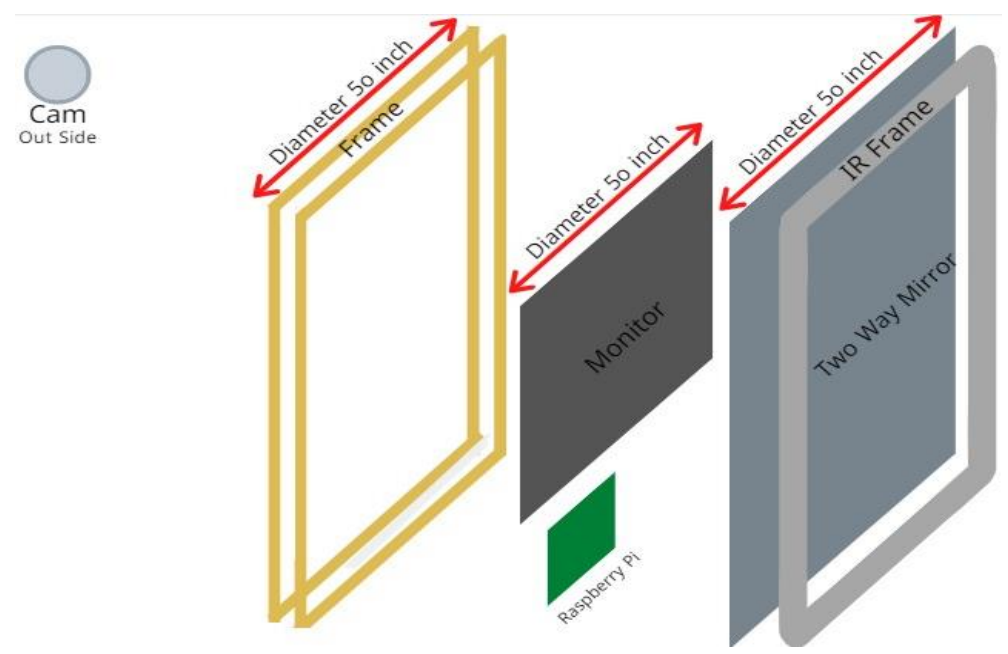

Figure 1. Hardware tools for smart mirror

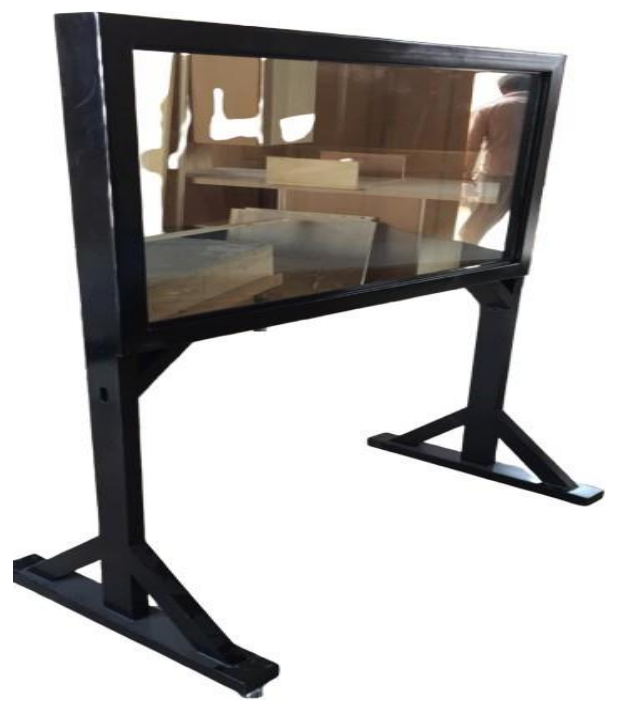

Figure 2. The wood frame with the two-way mirror

3. In the placing all together phase, the mirror is mounted on the LED monitor and it was connected to the control panel, which is the Raspberry Pi 4 model B that runs on Raspbian operating system, using an HDMI cable placed behind the mirror. After that, the Raspberry Pi was connected to a camera via Wi-Fi.

4. The IR frame was placed in front of the mirror to provide a touch interface and it was connected to the Raspberry Pi via USB port as shown in Figure 3.

5. Finally, as shown in Figure 4, the user interfaces of the system were implemented using Python programming language with the help of various packages and APIs such as OpenWeatherMap Weather API, which was used to get the climatic conditions of a particular location based on the latitude and longitude. 


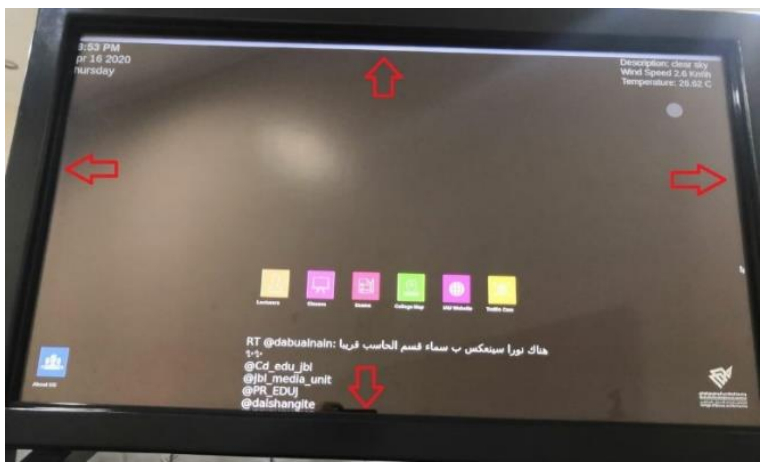

Figure 3. After putting the IR frame

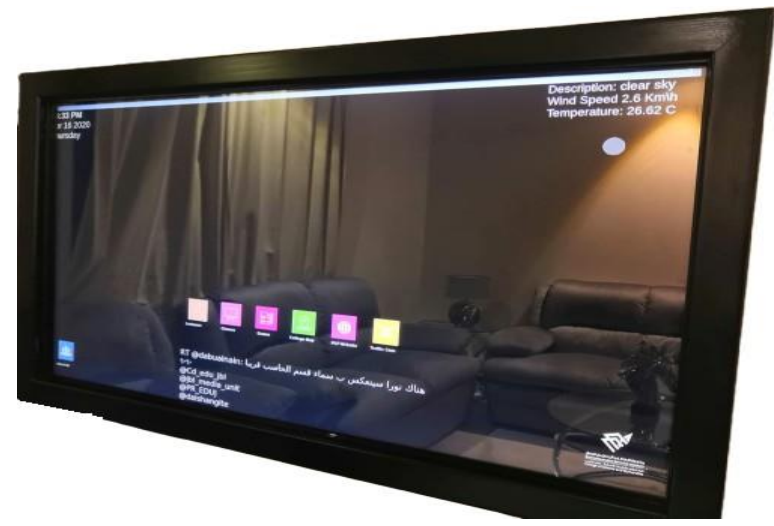

Figure 4. Final implementation

\subsection{Smart mirror as an information desk system}

The smart mirror works as an information desk system to provide the users with the suitable answers for their inquiries. In addition, the system could help the users with some other features such as navigation, web browsing and traffic monitoring. The developed system is user-friendly. The interfaces design is simple and focuses on the ease of use. The proposed system interfaces transfigure into the designed interfaces. It shows how the user experience (UX) design process will form the Wire-frames which are the proposed interfaces. After that, the user interface (UI) design process starts with taking the final UX prototypes and user flows to make it visually appealing for the end user. The designed UI determines what users do within a product. A combination of these two processes is needed to make a pleasing experience with consistent images and well laid out typography. The following subsections show the output.

\subsubsection{Main interface}

The main interface is shown in Figure 5 with the current time, date and weather of Jubail Industrial city in Saudi Arabia. The weather information and icon are fetched from the OpenWeatherMap online service so they could be updated automatically. This weather API is connected to our system Using unique user API key with Requests and Urllib python package. Furthermore, the main functions buttons are listed beside each other in the middle of the interface. Each button leads to a sub interface with one of the main functions. Moreover, the main functions of the system are divided between querying about the lecturers, courses and exams information. In addition, showing the university map, the university's website and the traffic Cam streaming. The university tweets stream is shown right under these icons. This tweets stream is fetched from Twitter developer's page using Twitter authentication API keys with Urllib and Twitter packages. The university logo is presented at the lower right corner.

\subsubsection{Lecturers query interface}

In this interface, the user can inquire about the lecturer's information such as the lecturer name, email, office number and hours. A query can be made by selecting the required lecturer name from a drop down list. Using this way will achieve the recognition rather than recall usability heuristic for a better user 
experience. Then, the lecturer information will be presented as an information card. Figure 6 shows the query results after execution. All sub interfaces contains a home button at the lower left corner to get back to the main interface.

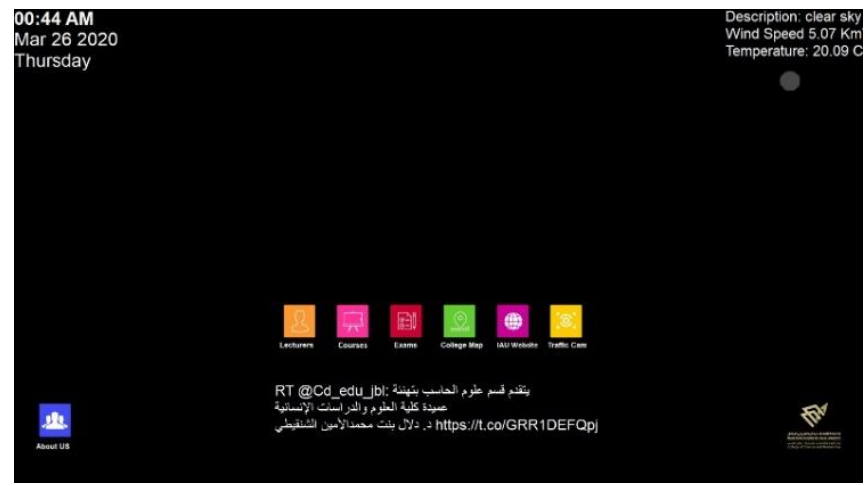

Figure 5. The main interface

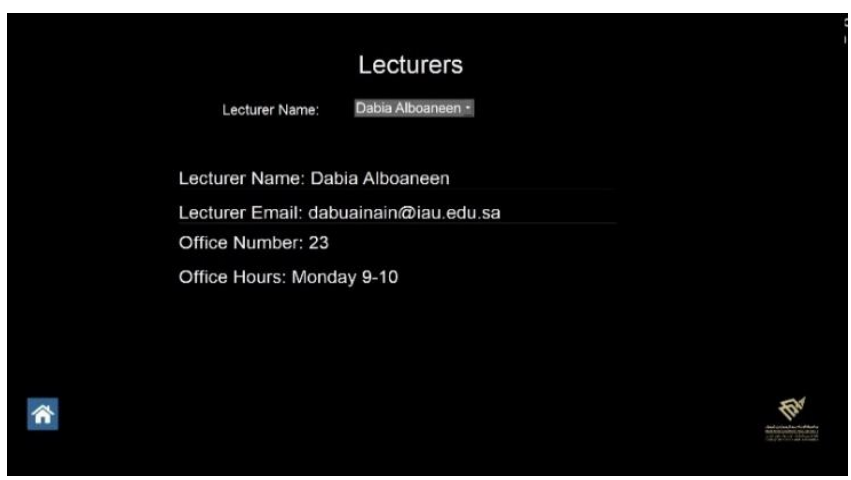

Figure 6. The lecturers interface after the query execution

\subsubsection{Courses query interface}

This interface is used to make queries about the courses information such as course Id, name, section ID, day and time. In addition, information about the course lecturers, hall ID and the wing number. It contains two drop down lists to select the desired course with a student specific section. The error prevention usability heuristic is applied in this interface in which the section list is disabled until the user chooses a course name first. In addition, the recognition rather than recall heuristic is also applied so the user does not have to recall the course name from the short-term memory. Figure 7 shows the query results after execution.

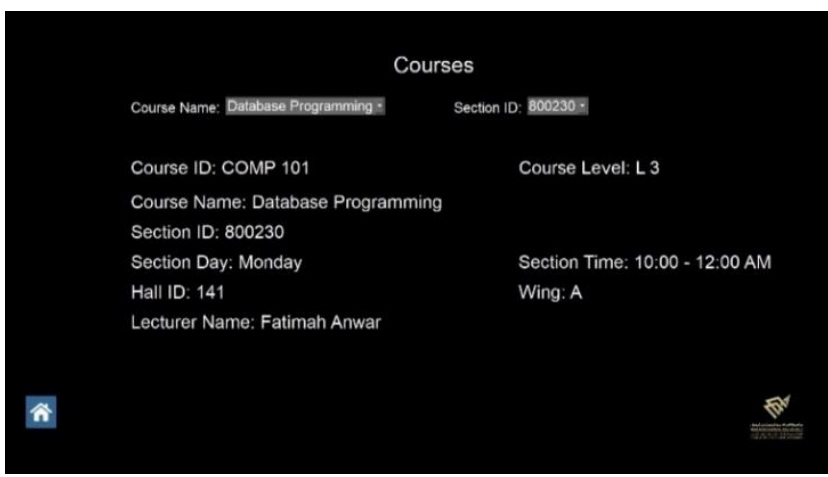

Figure 7. The courses interface after the query execution 


\subsubsection{Exams query interface}

In the same way, the user can inquire about the exams information using the course name through a drop down list. After the query execution, the result will be an exam information card as shown in Figure 8 . It contains the exam name, halls IDs, date and time that will be shown on the interface.

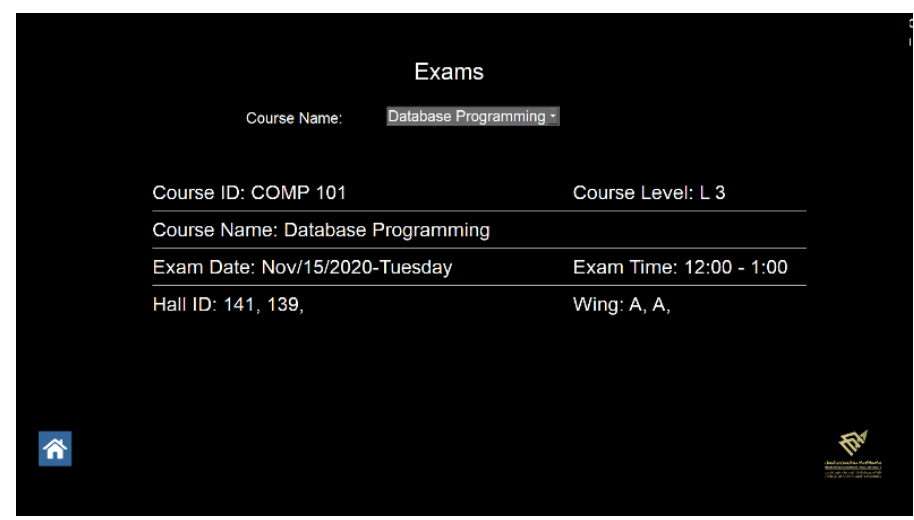

Figure 8 . The exams interface after the query execution

\subsubsection{University map interface}

In this interface, a 3D module of the university's first floor plan is presented. The user can choose to display the module in one of three view moods, which are 2D, top view, or 3D mood. Figure 9 shows the map in the $3 \mathrm{D}$ view mood.

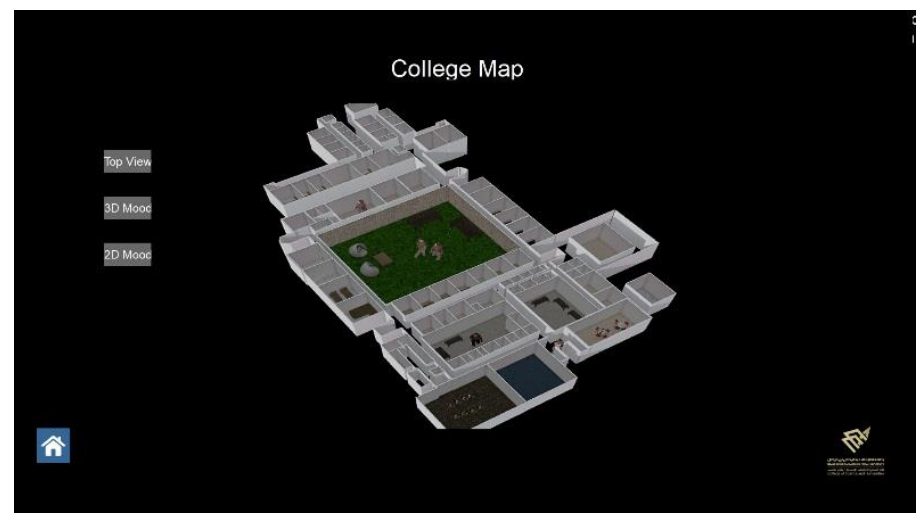

Figure 9. The university map interface in 3D mood

\subsubsection{The website interface}

This interface works as an impeded web browser in the system. It presents the official university's website as shown in Figure 10. This web browser is connected to the system using Webbrowser python library. Furthermore, the users can use this interface to view the university's latest news or access their accounts to use the services provided by the university's website. An onscreen virtual keyboard is used for any text entry such as the user's username and password. The keyboard is embedded in the browser, each pushed button represents a value either it was a letter or a number.

\subsubsection{Traffic cam interface}

An outdoor camera is connected to the system via Wi-Fi. The output will be a live stream video of the main entrance traffic. Figure 11 shows the video presented on the screen after the user opens the video streaming. The user can monitor the traffic and freeze the image at any time. This feature was not made for security purposes. The user can open and close the streaming since it is only a traffic monitoring cam. In this way, we can conserve the resources and energy even more. 


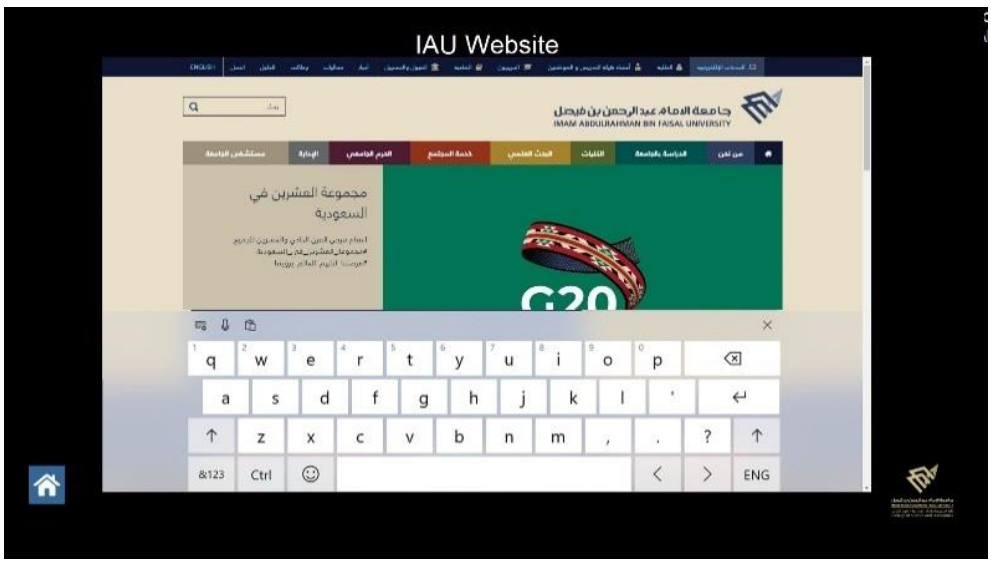

Figure 10. The website interface

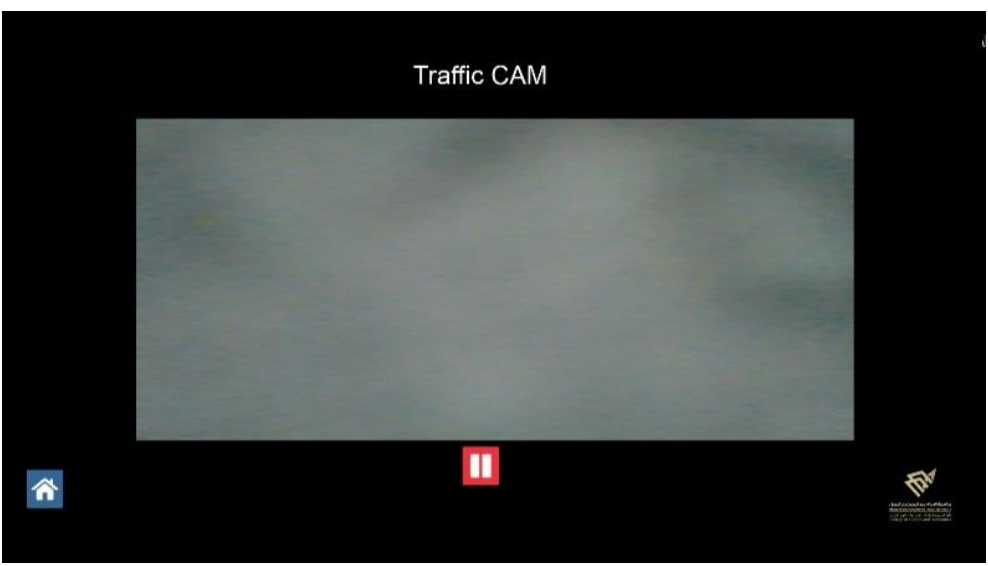

Figure 11. The traffic Cam interface after execution

\subsection{Smart mirror as a voice assistant}

At the beginning, there is a small process that keeps listening to detect a customized wake word from the continuous audio stream. The assistant processes this word as a password to start processing the voice commands fetched from the mini microphone. In this system, the user can say the phrase "Hey Mirror" to activate the voice assistant and start the conversation. The voice assistant would randomly choose from the predetermined responds such as "I'm ready" or "How can I help you?". Its job starts with a speech-to-text (STT) conversion process to convert the user's voice commands. The Speech Recognition python library is used for natural language processing and to convert the speech into a text. An audio file will be created to record the user voice commands. After that, it will be converted into a text to be handled. This procedure is followed by a text-to-speech (TTS) conversion process to convert the system text responds into a speech. This process is executed using Google-text-to-speech (GTTS) python library to translate the text into a speech. All responds will be read aloud from the audio file through the screen speakers. These two processes together are representing a speech-to-speech conversion. In between these processes, the whole idea depends on natural language processing. Furthermore, the mirror responds with a number of greetings in replay to the user's greetings.

The voice assistant workflow is presented in Figure 12. The procedure begins with fetching the input sound as commands in a continues loop. The assistant waits for the user to enter the password "Hey mirror" to be activated. It basically converts that audio input into a written command to start processing. The assistant starts to fetch the input sounds again to execute the matching function of its commands. Once it is executed, the textual output will be converted into an audio to be played through the speakers. If there is not a matching function the assistant responses with "I do not get that" sentence in audio to let the user re-enter the command. 


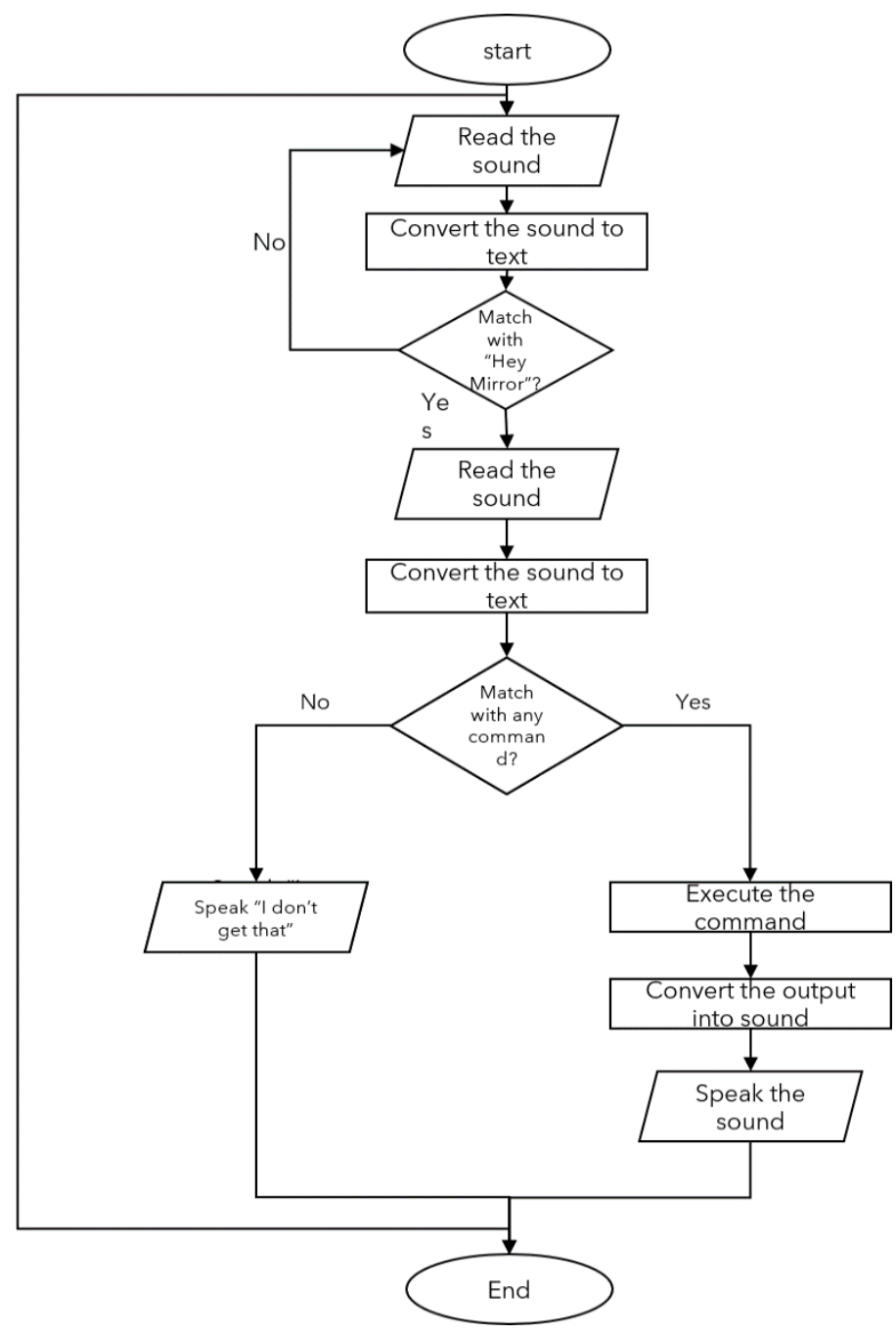

Figure 12. Flowchart of the voice assistant process

\section{CONCLUSION}

In this article, a smart information desk system is developed and installed on a Raspberry Pi 4 model B connected to a two-way mirror and IR touch frame. The system acts as a regular mirror with the ability to interact the users and answer their enquiries about academic information such as lecturers' office hours, halls numbers and courses timetables. In addition, voice recognition feature is utilized in which the system can receive these enquiries as voice commands.

Furthermore, the system provides other useful functionalities such as displaying general information along with the latest announcements from the university's Twitter account, and accessing the university's web- site. The system also displays real time images of the traffic at the university's main entrance using an external camera. Finally, it provides an interactive 3D map of the university building.

One aspect of the future work of smart mirrors is the manufacturing method. The mirror can be manufactured as a single layer, which will make it lighter in weight and easier to install as well. Moreover, a mobile application can be connected to the smart mirror's system to be used for keeping track of the user's inquiries history. Furthermore, novel speech recognition can be developed and facial recognition technology can be utilized in the implementation of smart mirrors for security purposes such as in processing the users images and verifying their identities.

\section{REFERENCES}

[1] D. A. Alboaneen et al., "Internet of things based smart mirrors: A literature review," in 2020 3rd International Conference on Computer Applications and Information Security (ICCAIS), 2020, pp. 1-6, doi: 10.1109/ICCAIS48893.2020.9096719. 
[2] S. S. Nathan, A. Sulaiman, A. A. Kamarulzaman, F. Tiera, and M. Berahim, "Brilliantreflect: smart mirror for smart life," International Journal of Electrical and Computer Engineering (IJECE), vol. 9, no. 3, pp. 1663-1668, 2019, doi: 10.11591/ijece.v9i3.pp1663-1668.

[3] Y. Sun, L. Geng, and K. Dan, "Design of smart mirror based on raspberry pi," in 2018 International Conference on Intelligent Transportation, Big Data and Smart City (ICITBS), 2018, pp. 77-80, doi: 10.1109/ICITBS.2018.00028.

[4] M. Ghazal, T. Al Hadithy, Y. Al Khalil, M. Akmal, and H. Hajjdiab, "A mobile-programmable smart mirror for ambient iot environments," in 2017 5th International Conference on Future Internet of Thingsand Cloud Workshops (FiCloudW), 2017, pp. 240-245, doi: 10.1109/FiCloudW.2017.106.

[5] A. S. A. Mohamed, M. N. Ab Wahab, S. S. Suhaily, and D. B. L. Arasu, "Smart mirror design powered by raspberry pi," in Proceedings of the 2018 Artificial Intelligence and Cloud Computing Conference, 2018, pp. 166-173, doi: 10.1145/3299819.3299840.

[6] S. Athira, F. Francis, R. Raphel, N. S. Sachin, S. Porinshu, and S. Francis, "Smart mirror: A novel framework for interactive display," in 2016 International Conference on Circuit, Power and Computing Technologies(ICCPCT), 2016, pp. 1-6, doi: 10.1109/ICCPCT.2016.7530197.

[7] J. R. Ding, C. L. Huang, J. K. Lin, J. F. Yang, and C. H. Wu, "Interactive multimedia mirror system design," IEEE Transactions on Consumer Electronics, vol. 54, no. 3, pp. 972-980, 2008, doi: 10.1109/TCE.2008.4637575.

[8] I. C. A. García, E. R. L. Salmón, R. V. Riega, and A. B. Padilla, "Implementation and Customization of a Smart Mirror through a Facial Recognition Authentication and a Personalized News Recommendation Algorithm," 2017 13th International Conference on Signal-Image Technology \& Internet-Based Systems (SITIS), 2017, pp. 35-39, doi: 10.1109/SITIS.2017.17.

[9] M. A. Hossain, P. K. Atrey, and A. E. Saddik, "Smart mirror for ambient home environment," 2007 3rd IET International Conference on Intelligent Environments, 2007, pp. 589-596, doi: 10.1049/cp:20070431.

[10] A. Benassi et al., "A wize mirror for lifestyle improvement," Stud Health Technol Inform, vol. 207, pp. 390-399, 2014.

[11] S. Colantonio et al., "Semeoticons-reading the face code of cardio-metabolic risk," in 2015 International Workshop on Computational Intelligence for Multimedia Understanding (IWCIM), 2015, pp. 1-5, doi: 10.1109/IWCIM.2015.7347092.

[12] S. Colantonio et al., "A smart mirror to promote a healthy lifestyle," BiosystemsEngineering, vol. 138, pp. 33-43, 2015, doi: 10.1016/j.biosystemseng.2015.06.008.

[13] Y. Andreu et al., "Wize mirror-a smart, multisensory cardio-metabolicrisk monitoring system," Computer Vision and Image Understanding, vol. 148, pp. 3-22, 2016, doi: 10.1016/j.cviu.2016.03.018.

[14] O. Erazo, J. A. Pino, R. Pino, A. Asenjo, and C. Fernández, "Magic Mirror for Neurorehabilitation of People with Upper Limb Dysfunction Using Kinect," 2014 47th Hawaii International Conference on System Sciences, 2014, pp. 2607-2615, doi: 10.1109/HICSS.2014.329.

[15] Y. Liu, J. Jia, J. Fu, Y. Ma, J. Huang, and Z. Tong, "Magic mirror: A virtual fashion consultant," in Proceedings of the 24th ACM international conference on Multimedia, 2016, pp. 680-683, doi: $10.1145 / 2964284.2970928$.

[16] L. Wang, R. Villamil, S. Samarasekera, and R. Kumar, "Magic mirror: A virtual handbag shopping system," in 2012 IEEE Computer Society Conference on Computer Vision and Pattern Recognition Work-shops, 2012, pp. 19-24, doi: 10.1109/CVPRW.2012.6239181.

[17] T. V. Nguyen and L. Liu, "Smart Mirror: Intelligent Makeup Recommendation and Synthesis," Proceedings of the 25th ACM international conference on Multimedia, Oct. 2017, pp. 1253-1254, doi: 10.1145/3123266.3127926.

[18] D. Saakes, H. S. Yeo, S. T. Noh, G. Han, and W. Woo, "Mirror Mirror: An On-Body T-shirt Design System," Proceedings of the 2016 CHI Conference on Human Factors in Computing Systems, May 2016, pp. 6058-6063, doi: $10.1145 / 2858036.2858282$.

[19] A. S. M. M. Rahman, T. T. Tran, S. A. Hossain and A. E. Saddik, "Augmented Rendering of Makeup Features in a Smart Interactive Mirror System for Decision Support in Cosmetic Products Selection," 2010 IEEE/ACM 14th International Symposium on Distributed Simulation and Real Time Applications, 2010, pp. 203-206, doi: 10.1109/DS-RT.2010.30.

[20] E. Iwabuchi, M. Nakagawa, and I. Siio, "Smart makeup mirror: computer-augmented mirror to aid makeup application," in International Conference on Human-Computer Interaction, 2009, pp. 495-503, doi: 10.1007/978-3-642-02583-9_54.

[21] M. Saba et al., "Smart mirror where i stand, who is the leanest in the sand?" in International Conference on Universal Access in Human-Computer Interaction, 2015, pp. 364-373, doi: 10.1007/978-3-319-20684-4_36.

[22] D. Besserer, J. Baurle, A Nikic, F. Honold, F. Schussel, and M. Weber, "Fitmirror: a smart mirror for positive affect in everyday user morning routines," Proceedings of the Workshop on Multimodal Analyses enabling Artificial Agents in Human-Machine Interaction, Nov. 2016, pp. 48-55, doi: 10.1145/3011263.3011265.

[23] T. Blum, V. Kleeberger, C. Bichlmeier, and N. Navab, "mirracle: An augmented reality magic mirror system for anatomy education," in 2012 IEEE Virtual Reality Workshops (VRW), 2012, pp. 115-116, doi: 10.1109/VR.2012.6180909.

[24] R. P. Yang, Z. T. Liu, L. D. Zheng, J. P. Wu, and C. C. Hu, "Intelligent mirror system based on facial expression recognition and color emotion adaptation mirror," in 2018 37th Chinese Control Conference (CCC), 2018, pp. 3227-3232, doi: 10.23919/ChiCC.2018.8483540.

[25] M. Fiala, "Magic mirror and hand-held and wearable augmentations," Virtual Reality Conference, 2007, pp. 251-254, doi: 10.1109/VR.2007.352493. 